\title{
CARLEMAN ESTIMATES WITH TWO LARGE PARAMETERS FOR SECOND ORDER OPERATORS AND APPLICATIONS TO ELASTICITY WITH RESIDUAL STRESS
}

Abstract. We derive Carleman type estimates with two large parameters for a general partial differential operator of second order. The weight function is assumed to be pseudo-convex with respect to the operator. We give applications to uniqueness and stability of the continuation of solutions and identification of coefficients for the Lamé system of dynamical elasticity with residual stress. This system is anisotropic and cannot be principally diagonalized, but it can be transformed into an "upper triangular" form. The use of two large parameters is essential for obtaining our results without smallness assumptions on the residual stress. In the proofs we use the classical technique of differential quadratic forms combined with a special partitioning of these forms and demonstrating positivity of terms containing highest powers of the second large parameter.

1. Introduction. We consider the general partial differential operator of second order

$$
A=\sum_{j, k=1}^{n} a^{j k} \partial_{j} \partial_{k}+\sum b^{j} \partial_{j}+c
$$

in a bounded domain $\Omega$ of the space $\mathbb{R}^{n}$ with the real-valued coefficients $a^{j k} \in C^{1}(\bar{\Omega}), b^{j}, c \in L^{\infty}(\Omega)$. The principal symbol of this operator is

$$
A(x, \zeta)=\sum a^{j k}(x) \zeta_{j} \zeta_{k} .
$$

We use the following convention and notations. Sums are over repeated indices $j, k, l, m=1, \ldots, n$. Let $\partial_{j}=\partial / \partial x_{j}, \partial=\left(\partial_{1}, \ldots, \partial_{n}\right), D=-i \partial$,

2000 Mathematics Subject Classification: 35R30, 35B60, 74B10.

Key words and phrases: inverse problems, continuation and prolongation of solutions, linear elasticity with initial stresses. 
$\alpha=\left(\alpha_{1}, \ldots, \alpha_{n}\right)$ is a multi-index with integer components, $\zeta^{\alpha}=\zeta_{1}^{\alpha_{1}} \cdots \zeta_{n}^{\alpha_{n}}$, $D^{\alpha}$ and $\partial^{\alpha}$ are defined similarly. $\nu$ is the outward normal to the boundary of a domain, $C, \kappa$ are generic constants (different at different places) depending only on the operator $A$, on the elastic parameters $\varrho, \lambda, \mu, R$, on the function $\psi$, and on the domain $\Omega$. In Theorems 1.2-1.6 we assume that $C, \kappa$ depend on $\Omega, \varrho, \lambda, \mu, R(; 2), \psi, \mathbf{u}_{0}, \mathbf{u}_{1}, M, \delta_{0}$. Any additional dependence will be indicated. We set $\Omega(\delta)=\Omega \cap\{\psi>\delta\}$. We recall that

$$
\|u\|_{(k)}(\Omega)=\left(\sum_{|\alpha| \leq k} \int_{\Omega}\left|\partial^{\alpha} u\right|^{2}\right)^{1 / 2}
$$

is the norm in the Sobolev space $H_{(k)}(\Omega)$ and \|\|$_{2}=\|\|_{(0)}$ is the $L^{2}$-norm.

A function $\psi$ is called pseudo-convex on $\bar{\Omega}$ with respect to $A$ if $\psi \in C^{2}(\bar{\Omega})$, $A(x, \nabla \psi(x)) \neq 0, x \in \bar{\Omega}$, and

$$
\begin{aligned}
\sum \partial_{j} \partial_{k} \psi(x) \frac{\partial A}{\partial \zeta_{j}} \frac{\partial A}{\partial \zeta_{k}}(x, \xi)+\sum\left(\frac{\partial A}{\partial \zeta_{k}} \partial_{k} \frac{\partial A}{\partial \zeta_{j}}-\partial_{k} A \frac{\partial^{2} A}{\partial \zeta_{j} \partial \zeta_{k}}\right) & \partial_{j} \psi(x) \\
& \geq K|\xi|^{2}
\end{aligned}
$$

for some positive constant $K$, for any $\xi \in \mathbb{R}^{n}$ and any point $x$ of $\bar{\Omega}$ provided

$$
A(x, \xi)=0, \quad \sum \frac{\partial A}{\partial \zeta_{j}}(x, \xi) \partial_{j} \psi(x)=0 .
$$

We will use the weight function

$$
\varphi=e^{\gamma \psi}
$$

and let $\sigma=\gamma \tau \varphi$ where $\gamma, \tau$ are some real numbers (large parameters).

TheOREM 1.1. Let $\psi$ be pseudo-convex with respect to $A$ in $\bar{\Omega}$. Then there are constants $C, C_{0}(\gamma)$ such that

$$
\int_{\Omega} \sigma^{3-2|\alpha|} e^{2 \tau \varphi}\left|\partial^{\alpha} u\right|^{2} \leq C \int_{\Omega} e^{2 \tau \varphi}|A u|^{2}
$$

for all $u \in C_{0}^{2}(\Omega)$ and $\alpha, \gamma, \tau$ with $|\alpha| \leq 1, C<\gamma$, and $C_{0}(\gamma)<\tau$.

In [2] this result (for $C^{\infty}$-coefficients) was stated without proof and in [3] there are incomplete proofs for isotropic hyperbolic equations.

In [14] it is shown that $\psi(x, t)=|x-a|^{2}-\theta^{2} t^{2}$ is pseudo-convex with respect to $A$ if the speed of propagation for $A$ is monotone in a certain direction. According to [15], $\psi(x, t)=d^{2}(x, a)-\theta^{2} t^{2}$ ( $d$ is the distance in the Riemannian metric determined by the elliptic part of $A$ ) is pseudo-convex provided some convexity type conditions are satisfied.

In [1], [3], [7] Carleman estimates with two large parameters were used to obtain results on unique continuation and controllability for thermoelasticity systems. 
In this paper we consider an elasticity system with residual stress $R$ [16]. In Theorems $1.2-1.6$ we let $x \in \mathbb{R}^{3}$ and $(x, t) \in \Omega \subset \mathbb{R}^{4}$. The residual stress is modeled by a symmetric second-rank tensor $R(x)=\left(r_{j k}(x)\right)_{j, k=1}^{3} \in C^{2}(\bar{\Omega})$ which is divergence free, $\nabla \cdot R=0$. Let $\mathbf{u}(x, t)=\left(u_{1}, u_{2}, u_{3}\right)^{\top}: \Omega \rightarrow \mathbb{R}^{3}$ be the displacement vector in $\Omega$. We introduce the operator of linear elasticity with residual stress

$$
\begin{aligned}
\mathbf{A}_{R} \mathbf{u}= & \varrho \partial_{t}^{2} \mathbf{u}-\mu \Delta \mathbf{u}-(\lambda+\mu) \nabla(\operatorname{div} \mathbf{u})-\nabla \lambda \operatorname{div} \mathbf{u} \\
& -2 \epsilon(\mathbf{u}) \nabla \mu-\operatorname{div}((\nabla \mathbf{u}) R),
\end{aligned}
$$

where $\varrho \in C^{1}(\bar{\Omega}), \lambda, \mu \in C^{2}(\bar{\Omega})$ are the density and Lamé parameters depending only on $x$, and $\epsilon(\mathbf{u})=\left(\frac{1}{2}\left(\partial_{i} u_{j}+\partial_{j} u_{i}\right)\right)$. Let

$$
\square(\mu ; R)=\partial_{t}^{2}-\sum_{j k} \frac{\mu \delta_{j k}+r_{j k}}{\varrho} \partial_{j} \partial_{k}
$$

and recall that $\sigma=\tau \gamma \varphi$.

THEOREM 1.2. Let $\psi$ be pseudo-convex with respect to the operators $\square(\mu ; R)$ and $\square(\lambda+2 \mu ; R)$ in $\bar{\Omega}$. Then there are constants $C, C_{0}(\gamma)$ such that

$$
\begin{aligned}
\int_{\Omega}\left(\sigma \left(\left|\nabla_{x, t} \mathbf{u}\right|^{2}+\left|\nabla_{x, t} \operatorname{div} \mathbf{u}\right|^{2}\right.\right. & \left.+\left|\nabla_{x, t} \operatorname{curl} \mathbf{u}\right|^{2}\right) \\
& \left.+\sigma^{3}\left(|\mathbf{u}|^{2}+|\operatorname{div} \mathbf{u}|^{2}+|\operatorname{curl} \mathbf{u}|^{2}\right)\right) e^{2 \tau \varphi} \\
\leq & C \int_{\Omega}\left(\left|\mathbf{A}_{R} \mathbf{u}\right|^{2}+\left|\nabla_{x}\left(\mathbf{A}_{R} \mathbf{u}\right)\right|^{2}\right) e^{2 \tau \varphi}
\end{aligned}
$$

for all $\mathbf{u} \in H_{0}^{3}(\Omega)$ and $\gamma>C, \tau>C_{0}$.

In [11] this result was obtained when $R$ is "small", without quantifying how small.

Let us consider the following Cauchy problem:

$$
\mathbf{A}_{R} \mathbf{u}=\mathbf{f} \quad \text { in } \Omega, \quad \mathbf{u}=\mathbf{g}_{0}, \partial_{\nu} \mathbf{u}=\mathbf{g}_{1} \quad \text { on } \Gamma \subset \partial \Omega,
$$

where $\Gamma \in C^{3}$. By a standard argument ([8, Section 3.2]) the Carleman estimate of Theorem 1.2 implies the following conditional Hölder stability estimate for (1.8) in $\Omega(\delta)$ (and hence uniqueness in $\Omega(0)$ ).

TheOREM 1.3. Suppose that all coefficients $\lambda, \mu, \varrho, R$ are in $C^{2}(\bar{\Omega})$. Let $\psi$ be pseudo-convex with respect to $\square(\mu ; R)$ and $\square(\lambda+2 \mu ; R)$ in $\bar{\Omega}$. Assume that $\bar{\Omega}(0) \subset \Omega \cup \Gamma$. Then there exist $C(\delta)>0$ and $\kappa(\delta) \in(0,1)$ such that the solution $\mathbf{u} \in H^{2}(\Omega)$ to (1.8) satisfies

$$
\begin{aligned}
\|\mathbf{u}\|_{(1)}(\Omega(\delta))+\|\operatorname{div} \mathbf{u}\|_{(1)}(\Omega(\delta))+\|\operatorname{curl} \mathbf{u}\|_{(1)} & (\Omega(\delta)) \\
& \leq C\left(F+M_{2}^{1-\kappa} F^{\kappa}\right),
\end{aligned}
$$

where $F=\|\mathbf{f}\|_{(1)}(\Omega(0))+\left\|\mathbf{g}_{0}\right\|_{(5 / 2)}(\Gamma)+\left\|\mathbf{g}_{1}\right\|_{(3 / 2)}(\Gamma)$ and $M_{2}=\|\mathbf{u}\|_{(2)}(\Omega)$. 
In Theorems 1.4-1.6 we assume that $\Omega=G \times(-T, T), \Gamma=\Gamma^{\prime} \times(-T, T)$, $\Gamma^{\prime} \subset \partial G$ and that the system (1.8) is t-hyperbolic. A sufficient condition of hyperbolicity is

$$
0 \leq \lambda, \quad 0<2 \mu I_{3}+R \quad \text { on } \bar{G} .
$$

This condition is satisfied when any eigenvalue of the matrix $R$ is strictly greater than $-2 \mu$; this happens when, for example, $\sum_{i, j=1}^{3} r_{i j}^{2}<4 \mu^{2}$ on $\bar{G}$. We use the conventional energy integral

$$
E(t ; \mathbf{u})=\int_{G}\left(\left|\partial_{t} \mathbf{u}\right|^{2}+|\nabla \mathbf{u}|^{2}+|\mathbf{u}|^{2}\right)(, t) .
$$

THEOREM 1.4. Suppose that $\lambda, \mu, \varrho, R$ are in $C^{2}(\bar{\Omega})$. Let $\psi$ be pseudoconvex with respect to $\square(\mu ; R)$, and $\square(\lambda+2 \mu ; R)$ in $\bar{\Omega}$. Assume that

$$
\psi<0 \quad \text { on } \bar{G} \times\{-T, T\}, \quad \psi \geq 0 \quad \text { on } G \times\{0\} .
$$

Let $\Gamma=\partial G \times(-T, T)$. Then there exists $C$ such that the solution $\mathbf{u} \in H^{2}(\Omega)$ to (1.8) satisfies

$$
\begin{aligned}
& E(t ; \mathbf{u})+E(t ; \nabla \mathbf{u}) \\
& \quad \leq C\left(\|\mathbf{f}\|_{(1)}(\Omega)+\left\|\mathbf{g}_{0}\right\|_{(5 / 2)}(\Gamma)+\left\|\mathbf{g}_{1}\right\|_{(3 / 2)}(\Gamma)\right)^{2}, \quad-T<t<T .
\end{aligned}
$$

Now we state results about identification of residual stress from additional boundary data.

Let $\mathbf{u}(; 1), \mathbf{u}(; 2)$ be solutions to

$$
\mathbf{A}_{R} \mathbf{u}=\mathbf{0} \quad \text { in } \Omega, \quad \mathbf{u}=\mathbf{u}_{0}, \partial_{t} \mathbf{u}=\mathbf{u}_{1} \quad \text { on } G \times\{0\},
$$

corresponding to sets of coefficients $R(; 1)$ and $R(; 2)$, respectively. We introduce the norm of the differences of the lateral Cauchy data

$$
\begin{aligned}
F_{c}=\sum_{\beta=2}^{4} & \left(\left\|\partial_{t}^{\beta}(\mathbf{u}(; 2)-\mathbf{u}(; 1))\right\|_{(5 / 2)}(\Gamma)\right. \\
& \left.+\left\|\partial_{t}^{\beta} \partial_{\nu}(\mathbf{u}(; 2)-\mathbf{u}(; 1))\right\|_{(3 / 2)}(\Gamma)\right) .
\end{aligned}
$$

By examining the equation (1.12), we can see that since the residual stress tensor is divergence free it appears in the equation without first derivatives. It turns out that a single set of Cauchy data is sufficient to recover the residual stress. To guarantee the uniqueness, we impose some nondegeneracy condition on the initial data $\left(\mathbf{u}_{0}, \mathbf{u}_{1}\right)$. More precisely, we assume that

$$
\operatorname{det} \mathbf{M} \geq \delta_{0} \quad \text { on } \bar{G},
$$

where

$$
\mathbf{M}=\left(\begin{array}{cccccc}
\partial_{1}^{2} \mathbf{u}_{0} & 2 \partial_{1} \partial_{2} \mathbf{u}_{0} & 2 \partial_{1} \partial_{3} \mathbf{u}_{0} & \partial_{2}^{2} \mathbf{u}_{0} & 2 \partial_{2} \partial_{3} \mathbf{u}_{0} & \partial_{3}^{2} \mathbf{u}_{0} \\
\partial_{1}^{2} \mathbf{u}_{1} & 2 \partial_{1} \partial_{2} \mathbf{u}_{1} & 2 \partial_{1} \partial_{3} \mathbf{u}_{1} & \partial_{2}^{2} \mathbf{u}_{1} & 2 \partial_{2} \partial_{3} \mathbf{u}_{1} & \partial_{3}^{2} \mathbf{u}_{1}
\end{array}\right)
$$


Note that $\mathbf{M}(x)$ is a $6 \times 6$ matrix-valued function. For example, one can check that $\mathbf{u}_{0}(x)=\left(x_{1}^{2}, x_{2}^{2}, x_{3}^{2}\right)^{\top}$ and $\mathbf{u}_{1}(x)=\left(x_{2} x_{3}, x_{1} x_{3}, x_{1} x_{2}\right)^{\top}$ satisfy (1.14) with $\delta_{0}=64$.

We first state the Hölder stability estimate for the coefficients in $\Omega(\delta)$.

We introduce the following bound:

$$
\left\|\partial_{t}^{\beta} \partial_{x}^{\alpha} \mathbf{u}(; j)\right\|_{\infty}(\Omega)<M \quad \text { when }|\alpha| \leq 3, \beta \leq 4 .
$$

TheOREM 1.5. Assume that $\psi$ is pseudo-convex with respect to the operators $\square(\mu ; R(; 2))$ and $\square(\lambda+2 \mu ; R(; 2))$ in $\bar{\Omega}$. Let the initial data $\left(\mathbf{u}_{0}, \mathbf{u}_{1}\right)$ satisfy (1.14) and $\mathbf{u}(; j), j=1,2$, satisfy (1.15). Assume that $\bar{\Omega}(0) \subset \Omega \cup \Gamma$. Then there exist constants $C=C(M, \delta)>0$ and $\kappa=\kappa(\delta) \in(0,1)$ such that

$$
\|R(; 1)-R(; 2)\|_{(0)}(\Omega(\delta)) \leq C F_{c}^{\kappa} .
$$

If $\Gamma$ is the whole lateral boundary and $T$ is sufficiently large, then a much stronger (and, in a certain sense, best possible) Lipschitz stability estimate holds.

Theorem 1.6. Assume that $\lambda, \mu, \varrho, R(; 1), R(; 2)$ are in $C^{2}(\bar{\Omega})$. Let $\psi$ be pseudo-convex with respect to $\square(\mu ; R(; 2))$ and $\square(\lambda+2 \mu ; R(; 2))$ in $\bar{\Omega}$. Assume that the condition (1.10) is satisfied. Let the initial data $\left(\mathbf{u}_{0}, \mathbf{u}_{1}\right)$ satisfy (1.14) and $\mathbf{u}(; j), j=1,2$, satisfy (1.15). Assume that $\bar{\Omega}(0) \subset \Omega \cup \Gamma$. Let $\Gamma=\partial G \times(-T, T)$. Then there exists $C=C(M)$ such that for $R(; 1)$, $R(; 2)$ satisfying the condition

$$
R(; 1)=R(; 2) \quad \text { on } \Gamma,
$$

one has

$$
\|R(; 2)-R(; 1)\|_{(0)}(G) \leq C F_{c} .
$$

The bound (1.15) follows from certain bounds on the initial and boundary value data and coefficients of the elasticity system with residual stress and from uniform hyperbolicity of this system. Indeed, one can augment (1.12) by the Dirichlet type boundary condition $\mathbf{u}=\mathbf{g}_{0}$ on $\partial G \times(-T, T)$. Assuming that $\mathbf{u}_{0} \in H^{9}(G), \mathbf{u}_{1} \in H^{8}(G), \mathbf{g}_{0} \in H^{9}(\partial G \times(-T, T)), \partial G \in C^{9}$, the $C^{5}(\bar{G})$-norms of $\varrho, \lambda, \mu, r_{j k}$ are bounded by $M$, appropriate compatibility conditions for $\mathbf{u}_{0}, \mathbf{u}_{1}, \mathbf{g}_{0}$ at $\partial G \times\{0\}$ are satisfied and the family of systems (1.12) is uniformly hyperbolic, from standard energy estimates and embedding theorems for Sobolev spaces one can derive the bound (1.15). Also one can use the stress boundary condition instead of the Dirichlet type condition.

Moreover, by using uniform pseudo-convexity and bounds on coefficients one can show that the constants $C, \kappa$ depend only on the bounds on the coefficients and the constant in the uniform pseudo-convexity condition. We will give the details in the forthcoming paper [10]. 
Now we give explicit sufficient conditions for pseudo-convexity of $\psi(x, t)$ $=|x-\beta|^{2}-\theta^{2} t^{2}$ when $\varrho, \lambda, \mu$ are constants and $R$ is small compared to $\varrho, \lambda, \mu$. Observe that our smallness condition is explicit compared to conditions in [11], [13]. Smallness of the residual stress is a natural assumption for applications in geophysics and material science.

We denote by $\|R\|$ the norm $\left(\sum_{j, k=1}^{3} r_{j k}^{2}\right)^{1 / 2}$ of a matrix $R=\left(r_{j k}\right)$. Let $\beta \in \mathbb{R}^{3}, D=\sup _{x \in G}|x-\beta|$ and $d=\inf _{x \in G}|x-\beta|$.

LEMma 1.7. Let $\theta, d_{1}$ be some numbers. Let $\varrho, \lambda, \mu$ be constants, the matrix $R$ be symmetric positive at any point of $\Omega$, and

$$
2 \mu \varrho \theta^{2}+3\|R+\mu I\|\|\nabla R\||x-\beta|<2 \mu^{2} \quad \text { on } \bar{\Omega} .
$$

Suppose that

$$
\theta^{2} T^{2}<d^{2}
$$

Then the function $\psi(x, t)=|x-\beta|^{2}-\theta^{2} t^{2}-d_{1}^{2}$ is pseudo-convex with respect to the anisotropic wave operator $A=\square(\mu ; R)$ in $\bar{\Omega}$.

The proof uses standard calculations to verify the pseudo-convexity condition (1.2) and is given in [9].

Assume that

$$
D^{2}<2 d^{2}
$$

Let $d_{1}=d$ and

$$
\frac{D^{2}-d^{2}}{\theta^{2}}<T^{2}<\frac{d^{2}}{\theta^{2}}
$$

Then the conditions (1.20) and (1.10) are satisfied. So if in addition (1.19) holds we have the conclusions of Theorems 1.4 and 1.6. The condition (1.21) can always be achieved by choosing $\beta$ at some distance from $G$. So the assumptions of Theorems 1.4 and 1.6 are satisfied when the observation time is sufficiently large and the residual stress is relatively small.

If, for some $d_{1}$,

$$
|x-\beta|^{2}-d_{1}^{2}<0 \quad \text { when } x \in \partial G \backslash \Gamma^{\prime}, \quad D^{2}-\theta^{2} T^{2}-d_{1}^{2}<0,
$$

then the assumptions of Theorems 1.3 and 1.5 are satisfied.

Carleman estimates were introduced by Carleman in 1939 to demonstrate uniqueness in the Cauchy problem for a first order system in $\mathbb{R}^{2}$ with nonanalytic coefficients. His idea turned out to be very fruitful and until now it dominates the field. In 1950-80s Carleman type estimates and unique continuation theorems have been obtained for wide classes of partial differential equations including general elliptic and parabolic equations of second order and some hyperbolic equations of second order. For an account of these results we refer to books [5], [8]. While there are still challenges for scalar partial differential operators, in many cases results are quite complete. The sit- 
uation with systems is quite different. No useful concept of pseudo-convexity is available for systems and Carleman estimates have been obtained only in very special cases. A general 1958 result of Calderón is applicable only to some elliptic systems of first order. Only recently there has been progress for classical isotropic dynamical Maxwell and elasticity systems [4]. This progress was achieved by using principal diagonalization and Carleman estimates for scalar hyperbolic equations. An important system of thermoelasticity cannot be prinicipally diagonalized, but it has "triangular" structure which allows one to obtain Carleman estimates and unique continuation by exploiting Carleman estimates for second order scalar operators with two large parameters [1], [7]. So far Carleman estimates with two large parameters were obtained only for elliptic, parabolic, and isotropic hyperbolic operators of second order [3]. Carleman estimates are also very useful in control theory (controllability and stabilization for initial boundary value problems) and inverse problems [8]. In particular, they were a main tool in the first proof of uniqueness and stability of all three elastic parameters in the dynamical Lamé system from two sets of boundary data [6]. Anisotropic systems have been studied only in some (important) particular cases, like small scalar perturbation of classical elasticity (elasticity with residual stress) in [11], [12], [13] where there are Carleman estimates, unique continuation and stability of identification of elastic coefficients for such systems.

In this paper we obtain Carleman estimates with two large parameters for general partial differential operators of second order (including as a particular case operators of hyperbolic type). Applying these estimates we obtain Carleman estimates, unique continuation results and stability of identification of the residual stress $R$ without smallness assumption on $R$, i.e. globally. We have to assume pseudo-convexity with respect to two scalar operators involving the residual stress.

We have stated our basic results above. In Section 2 we give complete proofs of crucial symbol bounds and outline the remaining parts of the proof of the fundamental Carleman estimate of Theorem 1.1 referring for complete proofs to [9]. In Section 3 we derive from this Carleman estimate a Carleman estimate for a system of elasticity with residual stress which explains the role of the two large parameters. In Section 4 we prove uniqueness and Hölder type stability estimate for identification of the six functions defining the residual stress from one set of special boundary measurements of displacement and stress. A crucial assumption is that the initial data are in a certain sense independent (which excludes the practically important zero initial conditions). This assumption enables us to use a modification of the 1981 method of Bukhgeim and Klibanov used by Imanuvilov and Yamamoto in 2002 for scalar equations. This modification was essential in handling inverse problems for isotropic elasticity in [6]. We observe that, if the initial 
data are zero, currently there are no uniqueness of identification results for the residual stress; moreover, most likely, no such results are true. Indeed, as is known, one cannot uniquely identify general (anisotropic) hyperbolic equations from all possible boundary measurements. In addition, even for the isotropic and scalar case, while there are complete uniqueness of identification results obtained by the method of boundary control of Belishev (see for example, [8]), stability of identification is very weak (of logarithmic type). This weak stability leads to poor results on numerical solution of inverse problems. Special nonzero initial data and pseudo-convexity assumptions imply much better Hölder and in some cases even best possible Lipschitz stability. Better stability promises a substantial improvement in numerical solution, which is crucial for applications.

2. Proof of Carleman estimates for scalar operators. In the following, $\zeta(\varphi)(x)=\xi+i \tau \nabla \varphi(x)$. We introduce the differential quadratic form

$$
\mathcal{F}(x, \tau, D, \bar{D}) v \bar{v}=|A(x, D+i \tau \nabla \varphi(x)) v|^{2}-|A(x, D-i \tau \nabla \varphi(x)) v|^{2} .
$$

This differential quadratic form is of order $(3,2)$, since the coefficients of the principal part of $A$ are real-valued. By Lemma 8.2.2 in Hörmander's book [5] there exists a differential quadratic form $\mathcal{G}(x, \tau, D, \bar{D})$ of order $(2,1)$ such that

$$
\int_{\Omega} \mathcal{G}(x, \tau, D, \bar{D}) v \bar{v}=\int_{\Omega} \mathcal{F}(x, \tau, D, \bar{D}) v \bar{v}
$$

its symbol is

$$
\mathcal{G}(x, \tau, \xi, \xi)=\left.\frac{1}{2} \sum \frac{\partial^{2}}{\partial x_{k} \partial \eta_{k}} \mathcal{F}(x, \tau, \zeta, \bar{\zeta})\right|_{\eta=0}, \quad \zeta=\xi+i \eta,
$$

where

$$
\begin{aligned}
\mathcal{F}(x, \tau, \zeta, \bar{\zeta})= & A(x, \zeta+i \tau \nabla \varphi) A(x, \bar{\zeta}-i \tau \nabla \varphi) \\
& -A(x, \zeta-i \tau \nabla \varphi) A(x, \bar{\zeta}+i \tau \nabla \varphi) .
\end{aligned}
$$

By using the formula for the symbol of $\mathcal{G}$ one can prove

LEMma 2.1. We have

$$
\begin{aligned}
\mathcal{G}(x, \tau, \xi, \xi)= & 2 \tau \sum \frac{\partial A}{\partial \zeta_{j}} \frac{\overline{\partial A}}{\partial \zeta_{k}} \partial_{j} \partial_{k} \varphi+2 \Im \sum \partial_{k} A \frac{\overline{\partial A}}{\partial \zeta_{k}} \\
& +2 \Im \sum A\left(\frac{\partial^{2} A}{\partial \zeta_{k} \partial x_{k}}-i \tau \overline{\frac{\partial^{2} A}{\partial \zeta_{j} \partial \zeta_{k}}} \partial_{j} \partial_{k} \varphi\right)
\end{aligned}
$$

where $A, \partial_{k} A, \ldots$ are taken at $(x, \zeta(\varphi)(x))$.

The following differentiation formulae follow from (1.4) and will be used in our proofs: 


$$
\partial_{j} \varphi=\gamma \varphi \partial_{j} \psi, \quad \partial_{j} \partial_{k} \varphi=\gamma \varphi \partial_{j} \partial_{k} \psi+\gamma^{2} \varphi \partial_{j} \psi \partial_{k} \psi .
$$

Using these formulae, from Lemma 2.1 by standard calculations we obtain

$$
\begin{aligned}
\tau^{-1} \mathcal{G}(x, \tau, \xi, \xi)= & \mathcal{G}_{1}(x, \tau, \xi, \xi)+\mathcal{G}_{2}(x, \tau, \xi, \xi) \\
& +\mathcal{G}_{3}(x, \tau, \xi, \xi)+\mathcal{G}_{4}(x, \tau, \xi, \xi)
\end{aligned}
$$

where

$$
\begin{aligned}
\mathcal{G}_{1}(x, \tau, \xi, \xi)= & 8 \gamma \varphi \sum a^{j m} a^{k l}\left(\xi_{m} \xi_{l}+\sigma^{2} \partial_{m} \psi \partial_{l} \psi\right) \partial_{j} \partial_{k} \psi, \\
\mathcal{G}_{2}(x, \tau, \xi, \xi)= & 4 \gamma \varphi \sum a^{l k} \partial_{k} a^{j m}\left(\sigma^{2} \partial_{j} \psi \partial_{m} \psi \partial_{l} \psi+2 \xi_{m} \xi_{l} \partial_{j} \psi-\xi_{j} \xi_{m} \partial_{l} \psi\right), \\
\mathcal{G}_{3}(x, \tau, \xi, \xi)= & 4 \gamma \varphi\left(2 \sum a^{k m} \partial_{j} a^{l j} \partial_{k} \psi \xi_{l} \xi_{m}\right. \\
& \left.-\sum a^{j k}\left(\partial_{m} a^{l m} \partial_{l} \psi+a^{l m} \partial_{l} \partial_{m} \psi\right)\left(\xi_{j} \xi_{k}-\sigma^{2} \partial_{j} \psi \partial_{k} \psi\right)\right), \\
\mathcal{G}_{4}(x, \tau, \xi, \xi)= & 4 \gamma^{2} \varphi\left(\left(2 \sum a^{j m} \xi_{m} \partial_{j} \psi\right)^{2}+2 \sigma^{2}\left(\sum a^{j m} \partial_{j} \psi \partial_{m} \psi\right)^{2}\right. \\
& \left.-\left(\sum a^{l m}\left(\xi_{l} \xi_{m}-\sigma^{2} \partial_{l} \psi \partial_{m} \psi\right)\right)\left(\sum a^{j k} \partial_{j} \psi \partial_{k} \psi\right)\right) .
\end{aligned}
$$

Observe that the terms of $\tau^{-1} \mathcal{G}$ with highest powers of $\gamma$ are collected in $\mathcal{G}_{4}$.

Proof of Theorem 1.1. First, make the substitution $u=e^{-\tau \varphi} v$. Obviously, $D_{k}\left(e^{-\tau \varphi} v\right)=e^{-\tau \varphi}\left(D_{k}+i \tau \partial_{k} \varphi\right) v$. Hence

$$
\sum a^{j k} D_{j} D_{k}\left(e^{-\tau \varphi} v\right)=\sum a^{j k} e^{-\tau \varphi}\left(D_{j}+i \tau \partial_{j} \varphi\right)\left(D_{k}+i \tau \partial_{k} \varphi\right) v .
$$

Accordingly, the bound (1.5) is transformed into

$$
\sum \int_{\Omega} \sigma^{3-2|\alpha|}\left|\partial^{\alpha} v\right|^{2} \leq C \int_{\Omega}|A(, D+i \tau \nabla \varphi) v|^{2} .
$$

LEMMA 2.2. Under the assumptions of Theorem 1.1, for any $\varepsilon_{0}$ there is C such that

$$
\begin{aligned}
\gamma \varphi(x)\left(2 K-\varepsilon_{0}\right)|\zeta(\varphi)(x)|^{2} & \\
& \leq \tau^{-1} \mathcal{G}(x, \tau, \xi, \xi)+\gamma \varphi(x) C \gamma^{2} \frac{|A(x, \zeta(\varphi)(x))|^{2}}{|\zeta(\varphi)(x)|^{2}}
\end{aligned}
$$

for all $\gamma>C, \xi \in \mathbb{R}^{n}$, and $x \in \bar{\Omega}$.

Proof. By homogeneity reasons we can assume $|\zeta(\varphi)|(x)=1$. In the proof we will use the fact that

$$
\begin{aligned}
A(x, \zeta(\varphi)(x)) & =\sum_{j, k=1}^{n} a^{j k}\left(\xi_{j} \xi_{k}-\sigma^{2} \partial_{j} \psi \partial_{k} \psi\right)+2 i \sum_{j, k=1}^{n} a^{j k} \sigma \xi_{j} \partial_{k} \psi \\
& =A(x, \xi)-\sigma^{2} A(x, \nabla \psi(x))+2 i \sigma \sum \frac{\partial A}{\partial \zeta_{j}}(x, \xi) \partial_{j} \psi(x)
\end{aligned}
$$


To show (2.7) we will use pseudo-convexity of $\psi$ and consider four possible cases.

CASE 1:

$$
\sigma=0, \quad A(x, \xi)=0, \quad \sum \frac{\partial A}{\partial \zeta_{j}}(x, \xi) \partial_{j} \psi(x)=0 .
$$

Then

$$
\sigma=0, \quad \sum a^{j k} \xi_{j} \xi_{k}=0, \quad \sum a^{j k} \xi_{j} \partial_{k} \psi=0,
$$

and from $(2.5)$ we obtain

$$
\begin{aligned}
\tau^{-1} \mathcal{G}(x, 0, \xi, \xi) \\
=2 \gamma \varphi \sum \partial_{j} \partial_{k} \psi 2 a^{j m} \xi_{m} 2 a^{k l} \xi_{l}+4 \gamma \varphi \sum a^{l k} \partial_{k} a^{j m}\left(2 \xi_{l} \xi_{m} \partial_{j} \psi-\xi_{j} \xi_{m} \partial_{l} \psi\right) \\
=2 \gamma \varphi \sum \partial_{j} \partial_{k} \psi \frac{\partial A}{\partial \zeta_{j}} \frac{\partial A}{\partial \zeta_{k}} \\
\quad+2 \gamma \varphi \sum\left(\left(\partial_{k} \frac{\partial A}{\partial \zeta_{j}}\right) \frac{\partial A}{\partial \zeta_{k}}-\left(\partial_{k} A\right) \frac{\partial^{2} A}{\partial \zeta_{j} \partial \zeta_{k}}\right) \partial_{j} \psi(x, \xi) \\
\geq 2 \gamma \varphi K
\end{aligned}
$$

by pseudo-convexity of $\psi(1.2)$.

CASE 2:

$$
\sigma<\delta, \quad\left|\gamma\left(A(x, \xi)-\sigma^{2} A(x, \nabla \psi(x))\right)\right|<\delta,
$$

where $\delta$ is a (small) positive number to be chosen later.

Using (2.5) as in Case 1, bounding the terms with $\sigma^{2}$ by $-C \gamma \varphi \delta$ and dropping the second (positive) term in $\mathcal{G}_{4}$ we obtain

$$
\begin{aligned}
& \tau^{-1} \mathcal{G}(x, \tau, \xi, \xi) \geq 2 \gamma \varphi \sum 2 a^{j m} \xi_{m} 2 a^{k l} \xi_{l} \partial_{j} \partial_{k} \psi-C \gamma \varphi \delta^{2} \\
& +4 \gamma \varphi \sum a^{l k} \partial_{k} a^{j m}\left(2 \xi_{l} \xi_{m} \partial_{j} \psi-\xi_{j} \xi_{m} \partial_{l} \psi\right) \\
& +4 \gamma \varphi 2 \sum \partial_{j} a^{l j} \xi_{l}\left(a^{k m} \partial_{k} \psi \xi_{m}\right) \\
& -4 \gamma \varphi \sum a^{j k}\left(\xi_{j} \xi_{k}-\sigma^{2} \partial_{j} \psi \partial_{k} \psi\right) \sum\left(\partial_{m} a^{l m} \partial_{l} \psi+a^{l m} \partial_{l} \partial_{m} \psi\right) \\
& +8 \gamma^{2} \varphi\left(\sum a^{j m} \xi_{m} \partial_{j} \psi\right)^{2} \\
& -\gamma \varphi\left(\gamma \sum a^{j k}\left(\xi_{j} \xi_{k}-\sigma^{2} \partial_{j} \psi \partial_{k} \psi\right)\right)\left(\sum a^{l m} \partial_{l} \psi \partial_{m} \psi\right) \\
\geq & 2 \gamma \varphi\left(\sum \partial_{j} \partial_{k} \psi 2 a^{j m} \xi_{m} 2 a^{k l} \xi_{l}+2 \sum a^{l k} \partial_{k} a^{j m}\left(2 \xi_{l} \xi_{m} \partial_{j} \psi-\xi_{j} \xi_{m} \partial_{l} \psi\right)\right) \\
& -C \gamma \varphi \delta+8 \gamma \varphi \gamma\left(\sum a^{j k} \xi_{j} \partial_{k} \psi\right)^{2},
\end{aligned}
$$

due to (2.8) and (2.10). 
Let us assume in addition to (2.10) that

$$
\left|\sum \frac{\partial A}{\partial \zeta_{j}}(x, \xi) \partial_{j} \psi(x)\right|<\delta .
$$

Then

$$
\begin{aligned}
h(x, \xi, \sigma) & =\sum \partial_{j} \partial_{k} \psi(x) 2 a^{j m}(x) \xi_{m} 2 a^{k l}(x) \xi_{l} \\
+ & 2 \sum a^{l k} \partial_{k} a^{j m}(x)\left(2 \xi_{l} \xi_{m} \partial_{j} \psi(x)-\xi_{j} \xi_{m} \partial_{l} \psi(x)\right) \geq K-\varepsilon(\delta)
\end{aligned}
$$

due to continuity arguments, compactness of the set

$$
M=\left\{(x, \xi, \sigma): x \in \bar{\Omega},|\xi|^{2}+\sigma^{2}|\nabla \psi(x)|^{2}=1\right\},
$$

and (2.10). Here $\varepsilon(\delta) \rightarrow 0$ as $\delta \rightarrow 0$.

Indeed, assuming the opposite of (2.13) we get a positive number $\varepsilon_{1}$ and a sequence $(x(k), \xi(k), \sigma(k)) \in M$ such that $h(x(k), \xi(k), \sigma(k)) \leq K-\varepsilon_{1}$ and (2.10), (2.12) hold with $\delta=k^{-1}$. Since $M$ is compact, (by extracting a subsequence if necessary) we may assume that $(x(k), \xi(k), \sigma(k)) \rightarrow(x, \xi, 0) \in M$ as $k \rightarrow+\infty$. By continuity $h(x, \xi, 0) \leq K-\varepsilon_{1}$. On the other hand, by the choice of the sequence, since $\gamma \geq 1,(x, \xi, 0)$ satisfies $(2.9)$. Hence by Case 1 , $h(x, \xi, 0) \geq K$ and we have a contradiction.

Because of (2.13) the right side in (2.11) is greater than

$$
\gamma \varphi(2 K-\varepsilon(\delta)-C \delta) \geq \gamma \varphi\left(2 K-\varepsilon_{0}\right) .
$$

Here we let $\delta<1 / C$, so that $\varepsilon(\delta)+C \delta<\varepsilon_{0}$. From now on we will fix such $\delta$ and denote it by $\delta_{0}$. We can choose $\delta_{0}$ to be dependent on the same parameters as $C$.

If

$$
\left|\sum \frac{\partial A}{\partial \zeta_{j}}(x, \xi) \partial_{j} \psi(x)\right| \geq \delta_{0},
$$

then using (2.10) with $\delta=\delta_{0}$ we conclude that the right side in (2.11) is greater than

$$
-C \gamma \varphi+8 \gamma \varphi \gamma \delta_{0}^{2} \geq \gamma \varphi 2 K
$$

when $\gamma>8^{-1} \delta_{0}^{-2}(C+2 K)$.

Finally, the condition (2.10) with $\delta=\delta_{0}$ implies (2.7).

To conclude the proof we observe that due to (2.8) in addition to (2.10) only the following cases 3 and 4 are possible.

CASE 3: $\sigma>\delta_{0}$ and $|\gamma \Re A(x, \zeta(\varphi)(x))|<\delta_{0}$. Using (2.5) as above we obtain

$$
\tau^{-1} \mathcal{G}(x, \tau, \xi, \xi) \geq-C \gamma \varphi(x)+\mathcal{G}_{4}(x, \tau, \xi, \xi)
$$


(by dropping the first (positive) term in $\mathcal{G}_{4}$, using that $\nabla \psi$ is noncharacteristic and bounding the last term in $\mathcal{G}_{4}$ from $(2.8)$ and $\delta_{0}$-smallness of $|\gamma \Re A|)$

$$
\geq-C \gamma \varphi(x)+8 C^{-1} \gamma^{2} \varphi \delta_{0}^{2} \geq 2 \gamma \varphi(x) K
$$

when we choose $\gamma>C^{2}$.

CASE 4: $\mid \gamma A\left(x, \zeta(\varphi)(x) \mid>\delta_{0}\right.$. From (2.5) we similarly have

$$
\begin{aligned}
& \tau^{-1} \mathcal{G}(x, \tau, \xi, \xi)+\gamma \varphi(x) C_{1}|\gamma A(x, \zeta(\varphi)(x))|^{2} \\
& \geq-C \gamma \varphi(x)-C \gamma^{2} \varphi|A(x, \zeta(\varphi)(x))|+\gamma \varphi C_{1}|\gamma A(x, \zeta(\varphi)(x))|^{2} \\
& \geq-C \gamma \varphi(x)-C \gamma \varphi(x)|\gamma A(x, \zeta(\varphi)(x))|+\gamma \varphi C_{1}|\gamma A(x, \zeta(\varphi)(x))|^{2} \\
& \geq-C \gamma \varphi(x)+C \gamma \varphi(x)|\gamma A(x, \zeta(\varphi)(x))|\left(\frac{C_{1}}{2 C}|\gamma A(x, \zeta(\varphi)(x))|-1\right) \\
&+\gamma \varphi(x) \frac{C_{1}}{2}|\gamma A(x, \zeta(\varphi)(x))|^{2} \\
& \geq-C \gamma \varphi(x)+C \gamma \varphi(x)|\gamma A(x, \zeta(\varphi)(x))|\left(\frac{C_{1} \delta_{0}}{2 C}-1\right)+\gamma \varphi(x) \frac{C_{1}}{2} \delta_{0}^{2} \\
& \geq K \gamma \varphi(x)
\end{aligned}
$$

when $C_{1}>2 C / \delta_{0}+(C+2 K) / 2 \delta_{0}^{2}$.

The proof is complete.

We fix $x_{0} \in \bar{\Omega}$, introduce the norm

$$
\left|\|v \mid\|_{-1}=\left(\int \frac{|\widehat{v}(\xi)|^{2}}{|\xi|^{2}+\tau^{2} \gamma^{2} \varphi^{2}\left(x_{0}\right)\left|\nabla \psi\left(x_{0}\right)\right|^{2}} d \xi\right)^{1 / 2}\right.
$$

and observe that

$$
\|v \mid\|_{-1} \leq C \tau^{-1}\|v\|_{2} .
$$

Lemma 2.3. There are a function $\varepsilon(\delta ; \gamma)$ convergent to 0 as $\delta \rightarrow 0$ for fixed $\gamma$ and a constant $C(\gamma)$ such that

$$
\begin{aligned}
\tau^{-1} \mid\left(\mathcal{G}\left(x_{0}, \tau, D, \bar{D}\right)-\right. & \mathcal{G}(, \tau, D, \bar{D}))\left.v \bar{v}\left|\leq \varepsilon(\delta ; \gamma) \sum_{|\alpha| \leq 1} \tau^{2-2|\alpha|}\right| \partial^{\alpha} v\right|^{2}, \\
\| \mid A\left(x_{0}, D+i \tau \nabla \varphi\left(x_{0}\right)\right) v- & A(, D+i \tau \nabla \varphi) v||_{-1}^{2} \\
\leq & \left(\varepsilon(\delta ; \gamma)+C(\gamma) \tau^{-1}\right) \sum_{|\alpha| \leq 1}\left(\gamma \tau \varphi\left(x_{0}\right)\right)^{2-2|\alpha|} \int\left|\partial^{\alpha} v\right|^{2}
\end{aligned}
$$

for all $v \in C_{0}^{2}\left(B\left(x_{0} ; \delta\right)\right)$.

The proof of this lemma is quite similar to the proof of Lemma 4.1 in [3]. It is given in detail in the forthcoming paper [9].

Now we continue the proof of Theorem 1.1. 
By using Parseval's identity,

$$
\left(\tau^{2}\left|\nabla \varphi\left(x_{0}\right)\right|^{2}\right)^{m-|\alpha|} \int\left|\partial^{\alpha} v\right|^{2} d x \leq(2 \pi)^{-n} \int|\zeta|^{2 m}(\varphi)\left(x_{0}\right)|\widehat{v}(\xi)|^{2} d \xi .
$$

Hence multiplying the inequality $(2.7)$ by $|\widehat{v}(\xi)|^{2}$ where $v \in C_{0}^{2}\left(\Omega_{\varepsilon}\right)$, and integrating over $\mathbb{R}^{n}$ yields

$$
\begin{aligned}
& C^{-1} \gamma \varphi\left(x_{0}\right) \sum_{|\alpha| \leq 1} \int\left(\gamma \tau \varphi\left(x_{0}\right)\right)^{2-2|\alpha|}\left|\partial^{\alpha} v\right|^{2} \\
& \leq \tau^{-1} \int \mathcal{G}\left(x_{0}, \tau, D, \bar{D}\right) v \bar{v}+\gamma \varphi\left(x_{0}\right) \gamma^{2} \int \frac{\left|A\left(x_{0}, \zeta(\varphi)\left(x_{0}\right)\right)\right|^{2}}{\left|\zeta(\varphi)\left(x_{0}\right)\right|^{2}}|\widehat{v}(\xi)|^{2} d \xi \\
& \leq \tau^{-1} \int \mathcal{G}\left(x_{0}, \tau, D, \bar{D}\right) v \bar{v}+\left.\gamma \varphi\left(x_{0}\right) \gamma^{2}\left\||| A\left(x_{0}, D+i \tau \nabla \varphi\left(x_{0}\right)\right) v\right\|\right|_{-1} ^{2} \\
& \leq \tau^{-1} \int \mathcal{G}(x, \tau, D, \bar{D}) v \bar{v}+\varepsilon(\delta ; \gamma) \sum_{|\alpha| \leq 1} \tau^{2-2|\alpha|} \int\left|\partial^{\alpha} v\right|^{2} \\
& \quad+\gamma \varphi\left(x_{0}\right) \gamma^{2}|| A(, D+i \tau \nabla \varphi) v \mid \|_{-1}^{2} \\
& \quad+\left(\varepsilon(\delta ; \gamma)+C(\gamma) \tau^{-2}\right) \sum_{|\alpha| \leq 1} \tau^{2-2|\alpha|} \int\left|\partial^{\alpha} v\right|^{2}
\end{aligned}
$$

for $v \in C_{0}^{2}\left(\Omega_{\varepsilon} \cap B\left(x_{0}, \delta\right)\right)$. Here we have used Lemma 2.3 and the elementary inequality $a^{2} \leq 2 b^{2}+2(b-a)^{2}$. Choosing $\delta>0$ small and $\tau$ large enough so that

$$
(2 C)^{-1} \gamma \varphi\left(x_{0}\right)\left(\gamma \tau \varphi\left(x_{0}\right)\right)^{2-2|\alpha|}>\left(\varepsilon(\delta ; \gamma)+C(\gamma) \tau^{-2}\right) \tau^{2-2|\alpha|}
$$

we absorb the second and fourth term on the right side of the inequality (2.16) to arrive at the inequality

$$
\begin{aligned}
& \sum_{|\alpha| \leq 1} \int\left(\gamma \tau \varphi\left(x_{0}\right)\right)^{3-2|\alpha|}\left|\partial^{\alpha} v\right|^{2} \\
& \quad \leq C \int \mathcal{G}(, \tau, D, \bar{D}) v \bar{v}+\tau \gamma \varphi\left(x_{0}\right) \gamma^{2}\|A(, D+i \tau \nabla \varphi) v\| \|_{-1}^{2} .
\end{aligned}
$$

As above by choosing large $\tau>C(\gamma)$ one can replace $\varphi\left(x_{0}\right)$ on the left side of this inequality by $\varphi$. Using $(2.1)$, (2.2) and the property (2.15) of the norm $\left.||||\right|_{-1}$ we conclude that

$$
\begin{aligned}
& \sum_{|\alpha| \leq 1} \int(\gamma \tau \varphi)^{3-2|\alpha|}\left|\partial^{\alpha} v\right|^{2} \\
& \quad \leq C\|A(, D+i \tau \nabla \varphi) v\|_{2}^{2}+C(\gamma) \tau^{-1}\|A(, D+i \tau \nabla \varphi) v\|_{2}^{2}
\end{aligned}
$$

for $v \in C_{0}^{2}\left(B\left(x_{0}, \delta\right)\right)$. Choosing $\tau>C(\gamma)$ we eliminate the second term on the right side. Now the bound (2.6) follows by a partition of unity argument. The proof is complete. 


\section{Proof of Carleman estimates for the elasticity system}

Lemma 3.1. Let $|\nabla \psi|>\delta_{0}>0$ on $\bar{\Omega}$. Then for a second order elliptic operator $A$ there are constants $C, C_{0}(\gamma)$ such that

$$
\gamma \int_{\Omega} \sigma^{4-2|\alpha|} e^{2 \tau \varphi}\left|\partial^{\alpha} v\right|^{2} \leq C \int_{\Omega} \sigma e^{2 \tau \varphi}|A v|^{2}
$$

for all $v \in C_{0}^{2}(\Omega),|\alpha| \leq 2, \gamma>C$ and $\tau>C_{0}(\gamma)$.

Proof. Apply the Carleman estimate in [3]:

$$
\sum_{|\alpha| \leq 2} \sqrt{\gamma}\left\|\sigma^{3 / 2-|\alpha|} e^{\tau \varphi} \partial^{\alpha} u\right\| \leq C\left\|e^{\tau \varphi} A(x, D) u\right\|
$$

to $u=\sigma^{1 / 2} v$.

Proof of Theorem 1.2. By using the standard substitution $(\mathbf{u}, v=\operatorname{div} \mathbf{u}$, $\mathbf{w}=$ curl $\mathbf{u}$ ) the system $\mathbf{A}_{R} \mathbf{u}=\mathbf{f}$ (with $\mathbf{A}_{R} \mathbf{u}$ given by (1.6)) can be reduced [11, Proposition 2.1] to a new system where the leading part is a special lower triangular matrix differential operator with the wave operators on the diagonal:

$$
\begin{aligned}
\square(\mu ; R) \mathbf{u} & =\frac{\mathbf{f}}{\varrho}+A_{1 ; 1}(\mathbf{u}, v), \\
\square(\lambda+2 \mu ; R) v & =\operatorname{div} \frac{\mathbf{f}}{\varrho}+\sum_{j k} \nabla\left(\frac{r_{j k}}{\varrho}\right) \cdot \partial_{j} \partial_{k} u+A_{2 ; 1}(\mathbf{u}, v, \mathbf{w}), \\
\square(\mu ; R) \mathbf{w} & =\operatorname{curl} \frac{\mathbf{f}}{\varrho}+\sum_{j k} \nabla\left(\frac{r_{j k}}{\varrho}\right) \times \partial_{j} \partial_{k} \mathbf{u}+A_{3 ; 1}(\mathbf{u}, v, \mathbf{w}),
\end{aligned}
$$

where $A_{j ; 1}$ are first order differential operators.

Applying Theorem 1.1 to each of the seven scalar differential operators forming the extended system (3.1) and summing up the resulting seven Carleman estimates, we get

$$
\begin{aligned}
\int_{\Omega}\left(\sigma\left|\nabla_{x, t} \mathbf{u}\right|^{2}\right. & \left.+\sigma\left|\nabla_{x, t} v\right|^{2}+\sigma\left|\nabla_{x, t} \mathbf{w}\right|^{2}+\sigma^{3}|\mathbf{u}|^{2}+\sigma^{3}|v|^{2}+\sigma^{3}|\mathbf{w}|^{2}\right) e^{2 \tau \varphi} \\
\leq & C \int_{\Omega}\left(\left|\mathbf{A}_{R} \mathbf{u}\right|^{2}+\left|\nabla\left(\mathbf{A}_{R} \mathbf{u}\right)\right|^{2}\right) e^{2 \tau \varphi}+C \int_{\Omega} \sum_{j, k=1}^{3}\left|\partial_{j} \partial_{k} \mathbf{u}\right|^{2} e^{2 \tau \varphi} \\
& +C \int_{\Omega}\left(|\nabla \mathbf{u}|^{2}+|\nabla u|^{2}+|\nabla \mathbf{w}|^{2}+|\mathbf{u}|^{2}+v^{2}+|\mathbf{w}|^{2}\right) e^{2 \tau \varphi} .
\end{aligned}
$$

By choosing $\tau>2 C$ the third integral on the right side can be absorbed by 
the left side. So we arrive at the inequality

$$
\begin{array}{r}
\int_{\Omega} Q\left(\sigma\left|\nabla_{x, t} \mathbf{u}\right|^{2}+\sigma\left|\nabla_{x, t} v\right|^{2}\right. \\
\left.+\sigma\left|\nabla_{x, t} \mathbf{w}\right|^{2}+\sigma^{3}|\mathbf{u}|^{2}+\sigma^{3}|v|^{2}+\sigma^{3}|\mathbf{w}|^{2}\right) e^{2 \tau \varphi} \\
\leq C \int_{\Omega}\left(\left|\mathbf{A}_{R} \mathbf{u}\right|^{2}+\left|\nabla\left(\mathbf{A}_{R} \mathbf{u}\right)\right|^{2}\right) e^{2 \tau \varphi}+C \int_{\Omega} \sum_{j, k=1}^{3}\left|\partial_{j} \partial_{k} \mathbf{u}\right|^{2} e^{2 \tau \varphi} .
\end{array}
$$

To eliminate the second order derivatives on the right side we need the second large parameter $\gamma$. By Lemma 3.1,

$$
\begin{aligned}
\gamma \int_{\Omega} \sum_{j, k=1}^{3}\left|\partial_{j} \partial_{k} \mathbf{u}\right|^{2} e^{2 \tau \varphi} & \leq C \int_{\Omega} \sigma|\Delta \mathbf{u}|^{2} e^{2 \tau \varphi} \leq C \int_{\Omega} \sigma\left(|\nabla v|^{2}+|\nabla \mathbf{w}|^{2}\right) e^{2 \tau \varphi} \\
& \leq C \int_{\Omega}\left(|\mathbf{f}|^{2}+|\nabla \mathbf{f}|^{2}\right) e^{2 \tau \varphi}+C \int_{\Omega}\left|\partial_{j} \partial_{k} \mathbf{u}\right|^{2} e^{2 \tau \varphi},
\end{aligned}
$$

where we have used the known identity $\Delta \mathbf{u}=\nabla v-\operatorname{curl} \mathbf{w}$ and (3.2). Choosing $\gamma>2 C$ we can see that the second order term on the right side is absorbed by the left side. This yields

$$
\gamma \int_{\Omega} \sum_{j, k=1}^{3}\left|\partial_{j} \partial_{k} \mathbf{u}\right|^{2} e^{2 \tau \varphi} \leq C \int_{\Omega}\left(|\mathbf{f}|^{2}+|\nabla \mathbf{f}|^{2}\right) e^{2 \tau \varphi} .
$$

So using again (3.2) we complete the proof of (1.7).

4. Hölder stability for the residual stress. In this section we prove Theorem 1.5. Let $\mathbf{u}(; 1)$ and $\mathbf{u}(; 2)$ satisfy (1.12) corresponding to $R(; 1)$ and $R(; 2)$, respectively. Set $\mathbf{u}=\mathbf{u}(; 2)-\mathbf{u}(; 1)$ and $\mathbf{F}=R(; 2)-R(; 1)=$ $\left(f_{j k}\right), j, k=1, \ldots, 3$. By subtracting equations $(1.12)$ for $\mathbf{u}(; 1)$ from the equations for $\mathbf{u}(; 2)$ we obtain

$$
\begin{aligned}
& \mathbf{A}_{R(; 2)} \mathbf{u}=\mathcal{A}(; \mathbf{u}(; 1)) \mathbf{F} \quad \text { on } \Omega, \text { where } \\
& \mathcal{A}(; \mathbf{u}(; 1)) \mathbf{F}=\sum_{j, k=1}^{3} f_{j k} \partial_{j} \partial_{k} \mathbf{u}(; 1)
\end{aligned}
$$

and

$$
\mathbf{u}=\partial_{t} \mathbf{u}=0 \quad \text { on } G \times\{0\} .
$$

Differentiating (4.1) in $t$ and using time-independence of the coefficients of the system, we get

$$
\mathbf{A}_{R(; 2)} \mathbf{U}=\mathcal{A}(; \mathbf{U}(; 1)) \mathbf{F} \quad \text { on } \Omega,
$$

where 


$$
\mathbf{U}=\left(\begin{array}{l}
\partial_{t}^{2} \mathbf{u} \\
\partial_{t}^{3} \mathbf{u} \\
\partial_{t}^{4} \mathbf{u}
\end{array}\right) \quad \text { and } \quad \mathbf{U}(; 1)=\left(\begin{array}{l}
\partial_{t}^{2} \mathbf{u}(; 1) \\
\partial_{t}^{3} \mathbf{u}(; 1) \\
\partial_{t}^{4} \mathbf{u}(; 1)
\end{array}\right)
$$

Since $\Gamma$ is noncharacteristic with respect to $\mathbf{A}_{R}$ we can uniquely solve the equation $\mathbf{A}_{R(; 2)} \mathbf{U}=\mathbf{0}$ on $\Gamma$ for $\partial_{\nu}^{2} \mathbf{U}$ in terms of $\mathbf{U}, \partial_{\nu} \mathbf{U}$ and their second and first tangential derivatives on $\Gamma$. Moreover,

$$
\left\|\partial_{\nu}^{2} \mathbf{U}\right\|_{(1 / 2)}(\Gamma) \leq C\left(\|\mathbf{U}\|_{(5 / 2)}(\Gamma)+\left\|\partial_{\nu} \mathbf{U}\right\|_{(3 / 2)}(\Gamma)\right) \leq C F_{c}
$$

due to definitions of $\mathbf{U}$ in (4.3) and of $F_{c}$ in (1.13).

By extension theorems for Sobolev spaces there exists $\mathbf{U}^{*} \in H^{3}(\Omega)$ such that

$$
\mathbf{U}^{*}=\mathbf{U}, \quad \partial_{\nu} \mathbf{U}^{*}=\partial_{\nu} \mathbf{U}, \quad \partial_{\nu}^{2} \mathbf{U}^{*}=\partial_{\nu}^{2} \mathbf{U} \quad \text { on } \Gamma,
$$

and

$$
\begin{aligned}
\left\|\mathbf{U}^{*}\right\|_{(3)}(\Omega) & \leq C\left(\|\mathbf{U}\|_{(5 / 2)}(\Gamma)+\left\|\partial_{\nu} \mathbf{U}\right\|_{(3 / 2)}(\Gamma)+\left\|\partial_{\nu}^{2} \mathbf{U}\right\|_{(1 / 2)}(\Gamma)\right) \\
& \leq C F_{c}
\end{aligned}
$$

due to (4.4).

We now introduce $\mathbf{V}=\mathbf{U}-\mathbf{U}^{*}$. Then

$$
\mathbf{A}_{R(; 2)} \mathbf{V}=\mathcal{A}(; \mathbf{u}(; 1)) \mathbf{F}-\mathbf{A}_{R(; 2)} \mathbf{U}^{*} \text { on } \Omega
$$

and

$$
\mathbf{V}=\partial_{\nu}(\mathbf{V})=\partial_{\nu}^{2}(\mathbf{V})=\mathbf{0} \quad \text { on } \Gamma
$$

To use the Carleman estimate (1.7) we need zero Cauchy data on $\partial \Omega(0)$. To create such data we introduce a cut-off function $\chi \in C^{2}\left(\mathbb{R}^{4}\right)$ such that $0 \leq \chi \leq 1, \chi=1$ on $\Omega(\delta / 2)$ and $\chi=0$ on $\Omega \backslash \Omega(0)$. By the Leibniz formula,

$$
\mathbf{A}_{R(; 2)}(\chi \mathbf{V})=\chi \mathbf{A}_{R(; 2)}(\mathbf{V})+\mathbf{A}_{1} \mathbf{V}=\chi \mathcal{A} \mathbf{F}-\chi \mathbf{A}_{R(; 2)} \mathbf{U}^{*}+\mathbf{A}_{1} \mathbf{V}
$$

and hence

$$
\nabla_{x} \mathbf{A}_{R(; 2)}(\chi \mathbf{V})=\nabla_{x}(\chi \mathcal{A} \mathbf{F})-\nabla_{x}\left(\chi \mathbf{A}_{R(; 2)} \mathbf{U}^{*}\right)+\mathbf{A}_{2} \mathbf{V}
$$

due to (4.7). Here (and below) $\mathbf{A}_{j}$ denotes a $j$ th order matrix differential operator with coefficients uniformly bounded by $C(\delta)$. By the choice of $\chi$, $\mathbf{A}_{j} \mathbf{V}=0$ on $\Omega(\delta / 2)$. Because of $(4.8), \chi \mathbf{V} \in H_{0}^{3}(\Omega)$, so we can apply to it the Carleman estimate (1.7) with fixed $\gamma$ to get

$$
\begin{aligned}
& \int_{\Omega} \tau\left(|\chi \mathbf{V}|^{2}+\left|\nabla_{x}(\chi \mathbf{V})\right|^{2}\right) e^{2 \tau \varphi} \\
& \leq C(\delta) \int_{\Omega}\left(|\mathbf{F}|^{2}+\left|\nabla_{x} \mathbf{F}\right|^{2}+\left|\mathbf{A}_{R(; 2)}\left(\mathbf{U}^{*}\right)\right|^{2}\right.
\end{aligned}
$$




$$
\begin{aligned}
& \left.+\left|\nabla_{x}\left(\mathbf{A}_{R(; 2)}\left(\mathbf{U}^{*}\right)\right)\right|^{2}\right) e^{2 \tau \varphi}+C \int_{\Omega \backslash \Omega(\delta / 2)}\left(\left|\mathbf{A}_{1} \mathbf{V}\right|^{2}+\left|\mathbf{A}_{2} \mathbf{V}\right|^{2}\right) e^{2 \tau \varphi} \\
\leq & C\left(\int_{\Omega}\left(|\mathbf{F}|^{2}+\left|\nabla_{x} \mathbf{F}\right|^{2}\right) e^{2 \tau \varphi}+F_{c}^{2} e^{2 \tau \Phi}+C(\delta) e^{2 \tau \delta_{1}}\right)
\end{aligned}
$$

where $\Phi=\sup _{\Omega} \varphi$ and $\delta_{1}=e^{\gamma \delta / 2}$. To get the last inequality we have used the bounds (4.6) and (1.15).

On the other hand, from (1.12), (4.1), (4.2) we have

$$
\varrho \partial_{t}^{2} \mathbf{u}=\sum f_{j k} \partial_{j} \partial_{k} \mathbf{u}(; 1), \quad \varrho \partial_{t}^{3} \mathbf{u}=\sum f_{j k} \partial_{t} \partial_{j} \partial_{k} \mathbf{u}(; 1)
$$

on $G \times\{0\}$. From now on we will consider the symmetric matrix-valued function $\mathbf{F}$ as a vector-valued function with components $\left(f_{11}, f_{12}, f_{13}, f_{22}, f_{23}, f_{33}\right)$. So using the definitions of $\mathbf{M}$ we obtain $\varrho\left(\partial_{t}^{2} \mathbf{u}, \partial_{t}^{3} \mathbf{u}\right)=\mathbf{M F}$ on $G \times\{0\}$, and from the condition (1.14) we have

$$
\mathbf{F}=\mathbf{M}^{-1}\left(\varrho\left(\partial_{t}^{2} \mathbf{u}, \partial_{t}^{3} \mathbf{u}\right)\right), \quad \nabla_{x} \mathbf{F}=\nabla_{x}\left(\mathbf{M}^{-1}\left(\varrho\left(\partial_{t}^{2} \mathbf{u}, \partial_{t}^{3} \mathbf{u}\right)\right)\right)
$$

on $G \times\{0\}$. Hence, by using (1.15),

$$
|\mathbf{F}|^{2}+\left|\nabla_{x} \mathbf{F}\right|^{2} \leq C \sum_{|\alpha| \leq 1, \beta=2,3}\left|\partial_{t}^{\beta} \partial_{x}^{\alpha} \mathbf{u}(, 0)\right|^{2} .
$$

Since $\chi(, T)=0$,

$$
\begin{gathered}
\int_{G}\left|\chi \partial_{t}^{\beta} \partial_{x}^{\alpha} \mathbf{u}(x, 0)\right|^{2} e^{2 \tau \varphi(x, 0)} d x=-\int_{0}^{T} \partial_{t}\left(\int_{G}\left|\chi \partial_{t}^{\beta} \partial_{x}^{\alpha} \mathbf{u}(x, t)\right|^{2} e^{2 \tau \varphi(x, t)} d x\right) d t \\
\leq \int_{\Omega} 2 \chi^{2}\left(\left|\partial_{t}^{\beta+1} \partial_{x}^{\alpha} \mathbf{u}\right|\left|\partial_{t}^{\beta} \partial_{x}^{\alpha} \mathbf{u}\right|+\tau\left|\partial_{t} \varphi\right|\left|\partial_{t}^{\beta} \partial_{x}^{\alpha} \mathbf{u}\right|^{2}\right) e^{2 \tau \varphi} \\
+2 \int_{\Omega \backslash \Omega(\delta / 2)}\left|\partial_{t}^{\beta} \partial_{x}^{\alpha} \mathbf{u}\right|^{2} \chi\left|\partial_{t} \chi\right| e^{2 \tau \varphi}
\end{gathered}
$$

where $\beta=2,3,|\alpha| \leq 1$. The right side does not exceed

$$
\begin{aligned}
& C\left(\int_{\Omega} \tau\left|\chi \partial_{x}^{\alpha} \mathbf{U}\right|^{2} e^{2 \tau \varphi}+C(\delta) \int_{\Omega \backslash \Omega(\delta / 2)}\left|\partial_{x}^{\alpha} \mathbf{U}\right|^{2} e^{2 \tau \varphi}\right) \\
& \quad \leq C\left(\int_{\Omega} \tau\left|\chi \partial_{x}^{\alpha} \mathbf{V}\right|^{2} e^{2 \tau \varphi}+C(\delta) \int_{\Omega \backslash \Omega(\delta / 2)}\left|\partial_{x}^{\alpha} \mathbf{U}\right|^{2} e^{2 \tau \varphi}+\tau \int_{\Omega}\left|\partial_{x}^{\alpha} \mathbf{U}^{*}\right|^{2} e^{2 \tau \varphi}\right)
\end{aligned}
$$

because $\mathbf{U}=\mathbf{V}+\mathbf{U}^{*}$.

Since $\chi=1$ on $G(\delta / 2), \varphi<\delta_{1}$ on $\Omega \backslash \Omega(\delta / 2)$ and on $G \backslash G(\delta / 2)$, and $\varphi<\Phi$ on $\Omega$, from these inequalities, (4.9), (4.6), and (1.15) we obtain

$$
\begin{aligned}
& \int_{G}\left|\partial_{t}^{\beta} \partial_{x}^{\alpha} \mathbf{u}\right|^{2}(, 0) e^{2 \tau \varphi(, 0)} \\
& \leq C\left(\int_{\Omega}\left(|\mathbf{F}|^{2}+\left|\nabla_{x} \mathbf{F}\right|^{2}\right) e^{2 \tau \varphi}+C(\delta) e^{2 \tau \delta_{1}}+\tau e^{2 \tau \Phi} F_{c}^{2}\right) .
\end{aligned}
$$


First we get this bound with $G(\delta / 2)$ instead of $G$ on the left side and then add to both sides of the inequality the integral over $G \backslash G(\delta / 2)$ which is bounded by $C(\delta) e^{2 \tau \delta_{1}}$ due to the bound (1.15) and the inequality $\varphi<\delta_{1}$ on $G \backslash G(\delta / 2)$. From (4.10) and (4.11) we obtain

$$
\begin{aligned}
\int_{G}\left(|\mathbf{F}|^{2}+\left|\nabla_{x} \mathbf{F}\right|^{2}\right) e^{2 \tau \varphi(, 0)} & \\
& \leq C\left(\int_{\Omega}\left(|\mathbf{F}|^{2}+\left|\nabla_{x} \mathbf{F}\right|^{2}\right) e^{2 \tau \varphi}+\tau e^{2 \tau \Phi} F_{c}^{2}+C(\delta) e^{2 \tau \delta_{1}}\right) .
\end{aligned}
$$

To eliminate the integral on the right side of (4.12) we observe that

$$
\begin{aligned}
& \int_{\Omega}\left(|\mathbf{F}|^{2}(x)+\left|\nabla_{x} \mathbf{F}\right|^{2}(x)\right) e^{2 \tau \varphi(x, t)} d x d t \\
& \quad=\int_{G}\left(|\mathbf{F}|^{2}(x)+\left|\nabla_{x} \mathbf{F}\right|^{2}(x)\right) e^{2 \tau \varphi(x, 0)}\left(\int_{-T}^{T} e^{2 \tau(\varphi(x, t)-\varphi(x, 0))} d t\right) d x .
\end{aligned}
$$

Due to our choice of $\varphi$ we have $\varphi(x, t)-\varphi(x, 0)<0$ when $t \neq 0$. Hence by the Lebesgue theorem the inner integral (with respect to $t$ ) converges to 0 as $\tau$ goes to infinity. By continuity of $\varphi$, this convergence is uniform with respect to $x \in G$. For $\tau>C$ we the integral over $\Omega(\delta / 2)$ on the right side of (4.12) can be absorbed by the left side, leading to the inequality

$$
\int_{\Omega(\delta)}|\mathbf{F}|^{2} e^{2 \tau \varphi(, 0)} \leq C\left(e^{2 \tau \Phi} F_{c}^{2}+C(\delta) e^{2 \tau \delta_{1}}\right) .
$$

Letting $\delta_{2}=e^{\gamma \delta} \leq \varphi$ on $\Omega(\delta)$ and dividing by $e^{2 \tau \delta_{2}}$ yields

$$
\begin{aligned}
\int_{\Omega(\delta)}|\mathbf{F}|^{2} & \leq C\left(\tau e^{2 \tau\left(\Phi-\delta_{2}\right)} F_{c}^{2}+e^{-2 \tau\left(\delta_{2}-\delta_{1}\right)}\right) \\
& \leq C(\delta)\left(e^{2 \tau \Phi} F_{c}^{2}+e^{-2 \tau\left(\delta_{2}-\delta_{1}\right)}\right)
\end{aligned}
$$

since $\tau e^{-2 \tau \delta_{2}}<C(\delta)$. To prove (1.16) it suffices to assume that $F_{c}<1 / C$. Then $\tau=-\log F_{c} /\left(\Phi+\delta_{2}-\delta_{1}\right)>C$ and we can use this $\tau$ in (4.14). Due to the choice of $\tau$,

$$
e^{-2 \tau\left(\delta_{2}-\delta_{1}\right)}=e^{2 \tau \Phi} F_{c}^{2}=F_{c}^{2\left(\delta_{2}-\delta_{1}\right) /\left(\Phi+\delta_{2}-\delta_{1}\right)}
$$

and from (4.14) we obtain (1.16) with $\kappa=\left(\delta_{2}-\delta_{1}\right) /\left(\Phi+\delta_{2}-\delta_{1}\right)$.

The proof of Theorem 1.5 is now complete.

Acknowledgments. This work was supported in part by the NSF grants DMS 04-05976, DMS 07-07734, and Emylou Keith and Betty Dutcher Distinguished Professorship at Wichita State University. 


\section{References}

[1] P. Albano and D. Tataru, Carleman estimates and boundary observability for a coupled parabolic-hyperbolic system, Electron. J. Differential Equations 2000, no. 22,15 pp.

[2] M. Eller, Carleman estimates with a second large parameter, J. Math. Anal. Appl. 249 (2000), 491-514.

[3] M. Eller and V. Isakov, Carleman estimates with two large parameters and applications, in: Contemp. Math. 268, Amer. Math. Soc., 2000, 117-137.

[4] M. Eller, V. Isakov, G. Nakamura and D. Tataru, Uniqueness and stability in the Cauchy problem for Maxwell's and elasticity systems, in: Collège de France Seminar, 14, Stud. Math. Appl. 31, Elsevier, 2002, 329-349.

[5] L. Hörmander, Linear Partial Differential Operators, Springer, 1963.

[6] O. Imanuvilov, V. Isakov and M. Yamamoto, An inverse problem for the dynamical Lamé system with two sets of boundary data, Comm. Pure Appl. Math. 56 (2003), 1366-1382.

[7] V. Isakov, On the uniqueness of the continuation for a thermoelasticity system, SIAM J. Math. Anal. 33 (2001), 509-522.

[8] - Inverse Problems for Partial Differential Equations, Springer, New York, 2006.

[9] V. Isakov and N. Kim, Carleman estimates with second large parameter for second order operators, in: Some Applications of Sobolev Spaces to PDEs, Int. Math. Ser. 9, Springer, 2008, 135-160.

[10] - - - Global uniqueness of residual stress from one set of boundary data, in preparation.

[11] V. Isakov, G. Nakamura and J.-N. Wang, Uniqueness and stability in the Cauchy problem for the elasticity system with residual stress, in: Contemp. Math. 333, Amer. Math. Soc., 2003, 99-113.

[12] V. Isakov, J.-N. Wang and M. Yamamoto, Uniqueness and stability of determining the residual stress by one measurement, Comm. Partial Differential Equations 23 (2007), 833-848.

[13] - - - - An inverse problem for a dynamical Lamé system with residual stress, SIAM J. Math. Anal. 39 (2007), 1328-1343.

[14] A. Khaidarov, Carleman estimates and inverse problems for second order hyperbolic equations, Math. USSR-Sb. 58 (1987), 267-277.

[15] I. Lasiecka, R. Triggiani and P. F. Yao, Inverse/observability estimates for second order hyperbolic equations with variable coefficients, J. Math. Anal. Appl. 235 (2000), $13-57$.

[16] C.-S. Man, Hartig's law and linear elasticity with initial stress, Inverse Problems 14 (1998), 313-320.

Department of Mathematics and Statistics

Wichita State University

Wichita, KS 67206, U.S.A.

E-mail: victor.isakov@wichita.edu

Received on 25.11.2007;

revised version on 22.10.2008 
